\title{
What Controls the Rate of Ultrafast Charge Transfer and Charge Separation Efficiency in Organic Photovoltaic Blends
}

Andreas C. Jakowetz, ${ }^{\dagger}$ Marcus L. Böhm, ${ }^{\dagger}$ Jiangbin Zhang, $^{\dagger}$ Aditya Sadhanala, ${ }^{\dagger}$ Sven Huettner, ${ }^{\ddagger}$ Artem A. Bakulin, ${ }^{\dagger} \S$ Akshay Rao, ${ }^{* \dagger}$ and Richard H. Friend ${ }^{*} \dagger$

${ }^{\dagger}$ Cavendish Laboratory, Department of Physics, University of Cambridge, J J Thomson Avenue, Cambridge CB3 0HE, United Kingdom

${ }^{\ddagger}$ Fakultät für Biologie, Chemie und Geowissenschaften, University Bayreuth, Universitätsstrasse 30, 95440 Bayreuth, Germany

${ }^{\S}$ Department of Chemistry, Imperial College London, London SW7 2AZ, United Kingdom

\section{Supporting Information}

ABSTRACT: In solar energy harvesting devices based on molecular semiconductors, such as organic photovoltaics (OPVs) and artificial photosynthetic systems, Frenkel excitons must be dissociated via charge transfer at heterojunctions to yield free charges. What controls the rate and efficiency of charge transfer and charge separation is an important question, as it determines the overall power conversion efficiency (PCE) of these systems. In bulk heterojunctions between polymer donor and fullerene acceptors, which provide a model system to understand the fundamental dynamics of electron transfer in molecular systems, it has been established that the first step of photoinduced electron transfer can be fast, of order $100 \mathrm{fs}$.

But here we report the first study which correlates differences in the electron transfer rate with electronic structure and morphology, achieved with sub-20 fs time resolution pump-probe spectroscopy. We vary both the fullerene substitution and donor/fullerene ratio which allow us to control both aggregate size and the energetic driving force for charge transfer. We observe a range of electron transfer times from polymer to fullerene, from 240 fs to as short as 37 fs. Using ultrafast electrooptical pump-push-photocurrent spectroscopy, we find the yield of free versus bound charges to be weakly dependent on the energetic driving force, but to be very strongly dependent on fullerene aggregate size and packing. Our results point toward the importance of state accessibility and charge delocalization and suggest that energetic offsets between donor and acceptor levels are not an important criterion for efficient charge generation. This provides design rules for next-generation materials to minimize losses related to driving energy and boost PCE.

\section{INTRODUCTION}

Like biological light-harvesting complexes (LHCs), in organic photovoltaic cells (OPVs) photon absorption leads to the formation of Frenkel exciton states. In order to dissociate these excitons, OPVs use a heterojunction between $\mathrm{p}$ - and n-type organic semiconductors (OSCs), where energetic offsets drive charge transfer $(\mathrm{CT}){ }^{1-9}$ This energetic offset is often referred to as the driving energy $(\Delta E)$. For OPV systems it is defined as the difference between the ionization potential of the donor $\left(\mathrm{IP}_{\mathrm{D}}\right)$, the electron affinity of the acceptor $\left(\mathrm{EA}_{\mathrm{A}}\right)$, and the energy of the generated exciton $\left(E_{\mathrm{ex}}\right)$. Figure 1 shows a scheme for the energy levels while the exciton energy is included in this one electron diagram for visualization purposes only. $\Delta E$ can be described by the difference between the LUMO levels of donor and acceptor.

Historically, electron transfer in OSCs and devices based on them have been described within a modified Marcus framework, which considers the tunnelling of point like charges. ${ }^{10}$ This description has also been extended to hybrid systems such as interfaces between molecular systems and metal oxides, which underpin dye-sensitized solar cells (DSSCs), and artificial photosynthetic systems. Moving beyond CT, another crucial question is what process leads to long-range charge separation. Electron transfer dissociates the exciton, giving an electron on the acceptor and hole on the donor. The hole and electron are still $0.5-1 \mathrm{~nm}$ apart, at which separation they should have significant Coulomb binding energy and form a charge-transfer state (CTS) ${ }^{10}$ A certain fraction of these CTSs will dissociate into free charges and a certain fraction will remain trapped and recombine to the ground state. The dissociation of these CTSs has often been described within a modified Onsager-Braun framework, within which thermal activation leads to hopping of charges within a disordered broadened density of states (DOS).

However, it has also been recognized that there are many shortcomings in applying these modified versions of Marcus

Received: May 18, 2016

Published: August 19, 2016 


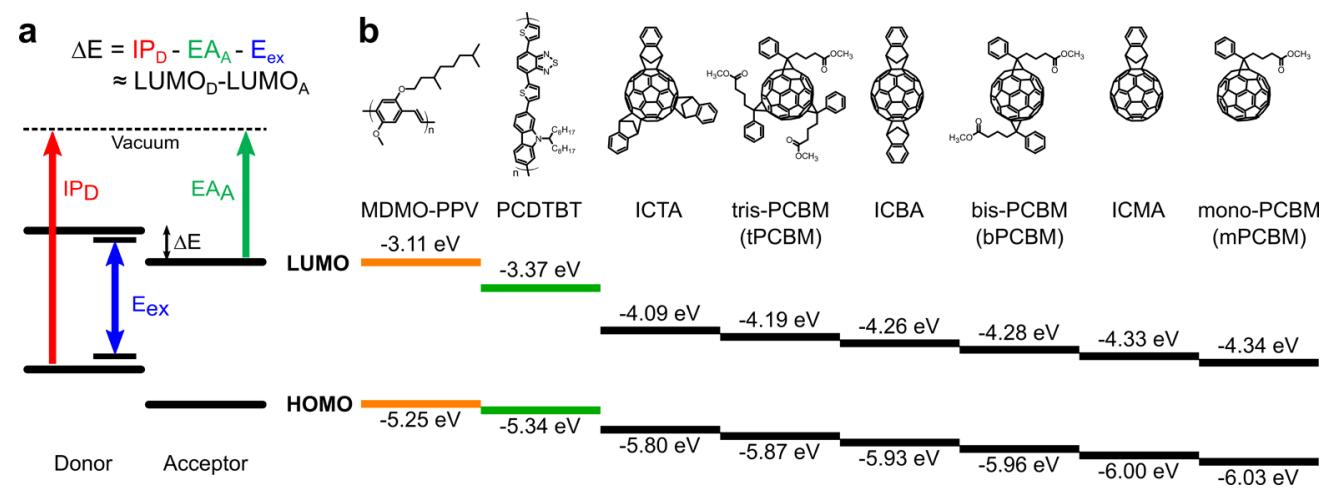

Figure 1. (a) Driving energy $(\Delta E)$ schematic diagram. $\Delta E$ is defined as the difference between the ionization potential of the donor (IP $P_{D}$, red), the electron affinity of the acceptor $\left(\mathrm{EA}_{\mathrm{A}}\right.$, green) and the energy of the initially generated exciton $\left(E_{\mathrm{ex}}\right.$, blue). (b) Chemical structures and energy levels of the polymer donors (color) and fullerene acceptors (black) materials used for this study. HOMO levels have been determined using ultraviolet photoelectron spectroscopy (UPS), and LUMO levels were determined by adding the band gap obtained by photothermal deflection spectroscopy (PDS, see Table S2.1) to the HOMO level.

theory to solid-state systems, for instance the availability of many states to couple to, rather than a single transition as in the Marcus framework. Indeed, many fundamental aspects of the $\mathrm{CT}$ process in the systems mentioned above, such as their ultrafast time scale $(<100 \mathrm{fs})$, electric field and temperature independence in some systems, cannot be accounted for within this framework. ${ }^{11}$ In the absence of a comprehensive theoretical framework for CT and charge separation, empirical design criterion have emerged within the literature to guide materials design, such as the need to have a LUMO level offset of 100$300 \mathrm{meV}$ between donor and acceptor, to provide sufficient driving energy for efficient CT and charge separation. . $^{8,12,13}$

Apart from energetics, the importance of blend morphology on device performance has been highlighted by several studies. For polymer/fullerene systems in particular it has been recognized that fullerene aggregation and the possibility of delocalization of electronic wave functions can play a crucial role. ${ }^{14-18}$ For instance, time-delayed collection field measurements, PL measurements and high-sensitivity EQE measurements have been used to study how charge generation efficiency varies with fullerene cluster size. Ultrafast studies have also hinted that in polymer/fullerene blends, charge delocalization on fullerenes may have a significant impact on charge separation efficiency. ${ }^{14,16,19}$ Recent experiments have shown that in some systems long-range electron-hole $(\mathrm{e}-\mathrm{h})$ separation occurs via an ultrafast "ballistic" process, leading to free charge generation in a few hundred femtoseconds, ${ }^{20-22}$ in a temperature independent process.

These experimental results have led to new theoretical models being proposed, which seek to address the role of delocalization of wave functions. These models are well summarized elsewhere. ${ }^{23}$ Delocalization is predicted to be very sensitive to fullerene cluster size and order, which would control the availability of states within the fullerene phase, and hence control both the rate of $\mathrm{CT}$ and $\mathrm{e}-\mathrm{h}$ separation. These models would also be less sensitive to LUMO offsets, $\Delta E$, than would be predicted within the standard Marcus/Onsager framework.

Which of these models, Marcus/Onsager or delocalized-wave functions/ballistic-transport, best describes the dynamics of OPVs, and under what energetic and morphological conditions, is a question of both fundamental and practical interest. Here, in model polymer/fullerene bulk heterojunction systems, we study the dependence of ultrafast electron transfer and charge separation efficiency on (1) driving energy $(\Delta E)$ and (2) state availability in the acceptor phase.

Studying the effect of $\Delta E$ on CT efficiency and $\mathrm{e}-\mathrm{h}$ pair separation is challenging and has previously been done only indirectly. ${ }^{24}$ This is because most studies of this nature vary the donor polymer against a fixed acceptor, usually a fullerene derivative. $^{25}$ Since different polymers can give rise to widely different blend morphologies, it is very difficult to deconvolute the effect of changes in $\Delta E$ from changes in morphology. Second, the effect of changes in $\Delta E$ is often studied by considering macroscopic quantities, such as EQEs, delayed charge collection or the charge yield at ns- $\mu$ s time scales, which can be affected by many parameters and not just the initial CT and separation process. ${ }^{26-28}$

In this study, we use pump-probe spectroscopy with sub-20 fs time-resolution and pump-push-photocurrent spectroscopy to directly measure the rates and yields for electron transfer and e-h pair separation. Using a variety of different fullerene-based acceptor materials enables us to sample a range of driving energies between -0.72 and $-1.23 \mathrm{eV}$. By changing the polymer/fullerene ratio, we are able to control the morphology of the phase-separated structure, and in particular, the presence and size of clusters of pure fullerenes, which occur preferentially at higher fullerene loading. We explore here how driving energy and fullerene cluster size control the rate of ultrafast CT and charge separation efficiency.

\section{RESULTS}

To tune the driving energy in OPVs, we consider two model systems. As donor species, we employ two different polymers, poly $\left[N-11^{\prime \prime}\right.$-henicosanyl-2,7-carbazole-alt-5,5-(4',7'-di-2-thienyl-2', $1^{\prime}, 3^{\prime}$-benzothiadiazole)] (PCDTBT) and poly[2-methoxy5-(3,7-dimethyloctyloxy)-1,4-phenylen]-alt-(vinylene) (MDMO-PPV), which are blended with six different fullerene derivatives, respectively: the Phenyl- $\mathrm{C}_{61}$-butyric acid methyl ester set mono- $\mathrm{PC}_{61} \mathrm{BM}(\mathrm{mPCBM})$, bis- $\mathrm{PC}_{61} \mathrm{BM}$ (bPCBM), tris- $\mathrm{PC}_{61} \mathrm{BM}$ (tPCBM), and the Indene- $\mathrm{C}_{60}$ mono-, bis-, and tris- adducts ICMA, ICBA, and ICTA at two different ratios (1:1 and 1:4 polymer/fullerene).

HOMO levels of pristine MDMO-PPV and PCDTBT as well as all fullerenes have been determined using ultraviolet photoelectron spectroscopy (UPS) on thin films of the pristine materials (see section S2, Figure S2.1). The corresponding LUMO levels have been obtained by adding the absorption 
band gap measured by photothermal deflection spectroscopy (PDS) to the HOMO energy, neglecting the exciton binding energy which would need to be added in order to obtain the precise LUMO level. The band gap for each material has been calculated as the center of a Gaussian fitted to the initial rise of the first derivative of each PDS absorption spectrum (Figure S2.2). A complete list of identified HOMO level deviations as well as the absorption band gaps can be found in Table S2.1. We note that despite neglecting the exciton binding energy and using the resulting slightly lower values for the LUMO energies, the energy differences between the different LUMO levels, as used in this study, are expected not to be influenced when assuming similar exciton binding energies in the different polymers and fullerenes.

These material combinations produce LUMO offsets in the range between -0.72 and $-1.23 \mathrm{eV}$. An overview of the materials and their respective highest-occupied molecular orbital (HOMO) and lowest-unoccupied molecular orbital (LUMO) levels is shown in Figure $1 \mathrm{~b}$. UV-vis and photoluminescence (PL) spectra for the polymers and monoPCBM, exemplarily for all fullerenes, can be found in Figure S1.1. We note that there is considerable variation in the LUMO levels reported for fullerenes in the literature. Taking values from the literature ${ }^{29,30}$ measured with the same technique (differential pulse voltammetry) gives LUMO offsets in the range between -0.63 and $0.01 \mathrm{eV}$, as shown in Figure S2.3. Thus, there is considerable ambiguity in the exact LUMO offsets for combinations of materials. But in this study, we are mainly interested in the trends observed as this offset is varied and not the absolute value. This trend is the same with either the values directly measured here, or by taking literature values. For the rest of this work, we will use values shown in Figure $1 \mathrm{~b}$.

The well-investigated polymers MDMO-PPV and PCDTBT were chosen as donor species since the electronic energy levels for these amorphous structures are likely to change little even when the fullerene loadings change. ${ }^{31}$ In addition, it is known that fullerenes are highly miscible in these polymers, with the fullerene intercalating between the polymer side chains. At low concentrations, the fullerenes are well dispersed throughout the polymer film. ${ }^{31-33}$ As the concentration of fullerene is increased and "excess" fullerene is added, fullerene aggregates are formed, which represents a pure fullerene phase. This means at higher fullerene loadings ( $50 \%$ and above studied here), there are no pure polymer domains within such films, which instead consist of areas of mixed polymer and fullerene and pure fullerene aggregates. Thus, excitons are always photogenerated close to fullerene sites at which they can undergo electron transfer. This is greatly beneficial from an experimental point of view as it means that on ultrafast time scales excitons do not have to diffuse before undergoing charge transfer.

Measuring the small-/wide-angle X-ray scattering (SAXS/ WAXS) spectra of the different materials and compositions provides a window on the packing of the fullerene in the films. The results (see section S3) show that monoadduct fullerenes form the largest aggregates, while adding more side groups disrupts packing and lowers aggregate size. Furthermore, increasing fullerene content leads to larger aggregates. Thus, the kind and amount of fullerene provide control over aggregate size and packing, which may have an impact on wave function delocalization. ${ }^{34}$

In order to measure the charge generation rates, we use ultrafast broadband pump-probe spectroscopy (PP). In this experiment, transmission $(T)$ of a material is probed using fast broadband laser pulses while exciting the sample with a pump pulse. Taking the difference of transmission spectra $(\Delta T)$ with and without excitation, yields spectral features of the excited states present in the material. By changing the time delay between pump and probe pulse, the temporal evolution of these excited state spectra can be obtained, resulting in PP maps like Figure 2a and $\mathrm{c}$.
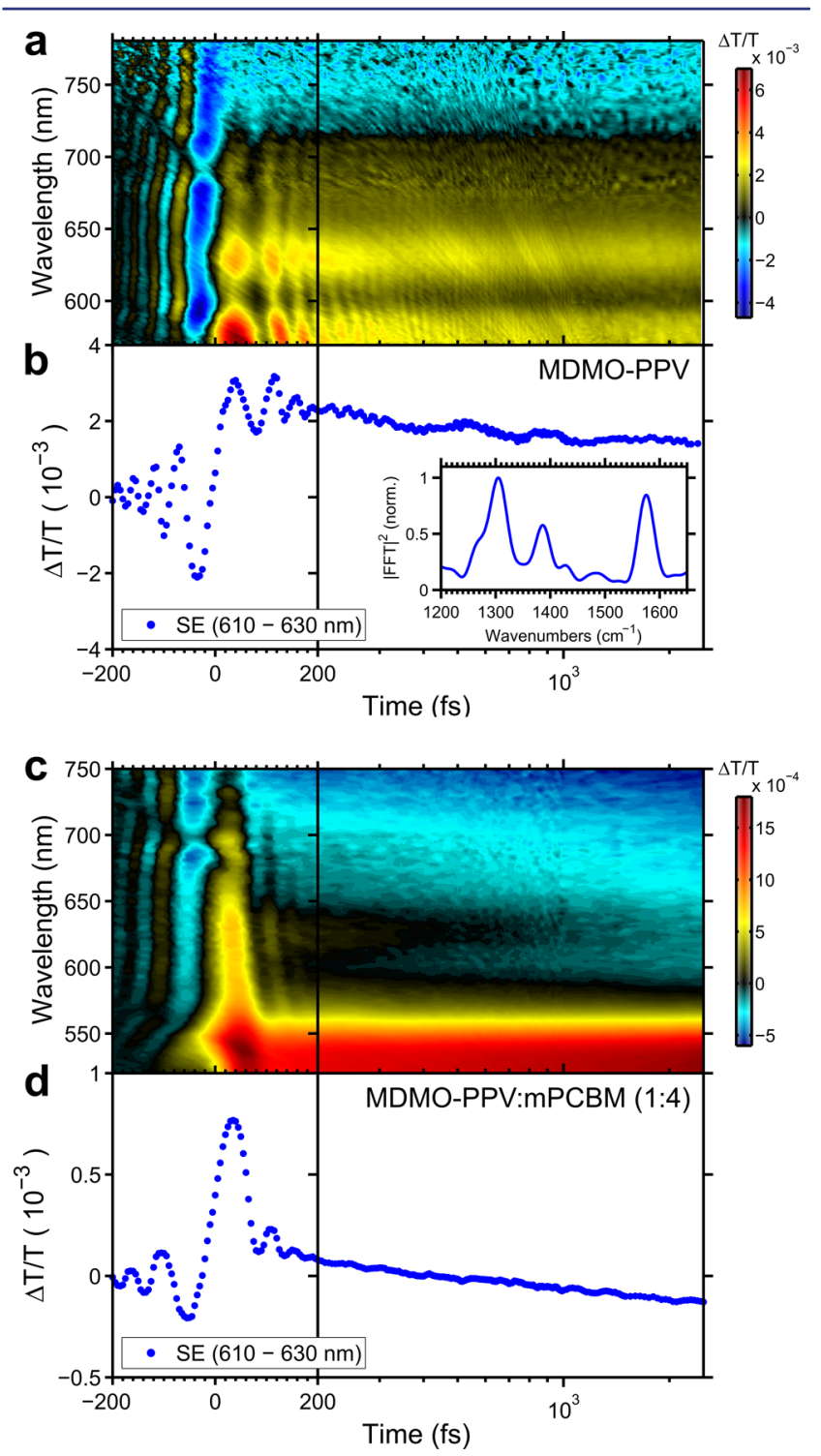

Figure 2. Pump-probe data $(\Delta T / T)$ for (a) pristine MDMO-PPV and (c) MDMO-PPV:mPCBM (1:4), acquired using 520-630 nm broad, sub-20 fs pulses with a fluence of $40 \mu \mathrm{J} / \mathrm{cm}^{2}$. (b, d) Corresponding SE kinetic traces averaged over $610-630 \mathrm{~nm}$. The inset in (b) contains the fast Fourier transformation of the oscillations on the corresponding kinetic after $t=0$ and with subtraction of the population background. The peaks at 1305 and $1575 \mathrm{~cm}^{-1}$ correspond to vibrational modes on MDMO-PPV with a period of 25.6 and 21.2 fs, respectively.

The samples were fabricated on ultrathin glass substrates $(130 \mu \mathrm{m})$ in order to reduce coherent artifacts due to the substrate and to reduce the amount of chirp added to the laser pulses. The 1:4 polymer/fullerene blends were measured with sub-20 fs pump pulses, and the 1:1 compositions were pumped with sub-40 fs pulses to minimize the impact of the coherent 
artifact on the data set. The pump pulses cover the spectral range between 520 to $630 \mathrm{~nm}$ which targets mostly the polymer absorption.

To study the charge carrier kinetics after the pump excitation we use broadband probe pulses in the range between 500-800 $\mathrm{nm}$, e.g. see Figure $2 \mathrm{a}$ and $\mathrm{c}$, which covers the spectral region of ground state bleach (GSB), stimulated emission (SE) and photoinduced absorption (PIA) for both polymers. We note that the spectral ranges for GSB and SE can be estimated from the respective spectra in steady-state UV-vis absorption and photoluminescence (see Figure S1.1). Their shape, however, can be different due to an overlapping negative PIA feature on the $\Delta T / T$ signal.

We focus on the SE signal, as its quenching provides a measure for the time scales of charge carrier generation from initially photogenerated excitons on the polymer. We choose to study the SE as it provides a relatively clean signal of the singlet exciton population. In contrast, both the singlet excitons and hole-polarons have broad PIAs in the near IR. As excitons are dissociated into holes, the spectral shape of this PIA changes but deconvolution of these intermixed features on fs time scales generates ambiguity. Data showing the rise of the hole polaron in the NIR (shown in Figure S4.3 along with fluence dependent data in Figure S4.4) are consistent with the dynamics extracted from the SE quenching. Also, although the excitation pulses are tuned to the polymer absorption, especially in the 1:4 composites there is some direct photoexcitation of the fullerenes which leads to hole transfer from the fullerene to the polymer. In order to minimize its impact on the data set, we measure the rate of SE quenching, rather than the charge population which contains contributions from both electron and hole transfer. We average the SE kinetics over a range of 610-630 nm for the MDMO-PPV blends and over 720-740 $\mathrm{nm}$ for the PCDTBT blends which represent the centers of the respective stimulated emission signals. Figure $2 \mathrm{a}$ and $\mathrm{b}$ show the PP spectra and SE kinetics of pristine MDMO-PPV as a reference, exhibiting long-lived SE and showing vibrational oscillations on top of the PP signal over the first several hundred femtoseconds. The inset of Figure $2 \mathrm{~b}$ shows the fast Fourier transformation of these oscillations. The peaks at 1305 and $1575 \mathrm{~cm}^{-1}$ correspond to vibrational modes on MDMOPPV with a period of 25.6 and 21.2 fs, respectively, which can only be set off with ultrafast excitation pulses shorter than the oscillation period of the mode, in this case pules shorter than 20 fs. $^{35}$

Comparing these kinetics with the SE decay in the corresponding MDMO-PPV:PCBM blend (1:4; see Figure 2c and d) reveals rapid exciton quenching due to electron transfer to the fullerene on a time scale (exponential lifetime) of $37 \mathrm{fs}$. The SE kinetics are fitted with a monoexponential function over the first $500 \mathrm{fs}$ for 1:1 blends and over the first $300 \mathrm{fs}$ for the 1:4 compositions:

$$
\mathrm{SE}(t)=\mathrm{SE}_{\text {off }}+\mathrm{SE}_{0} \exp \left(-k_{\mathrm{CG}} t\right)
$$

The obtained time constants $k_{\mathrm{CG}}$ are a figure of merit for the respective $\mathrm{CT}$ rates.

Figure 3 shows the charge generation rates $k_{\mathrm{CG}}$ for MDMOPPV:fullerene (red) and PCDTBT:fullerene (blue) blend ratios of 1:1 (hollow symbols) and 1:4 (solid symbols) as a function of energetic offset between donor and acceptor (i.e., driving energy; $\Delta$ LUMO). The dashed (1:4 ratio) and dotted-dashed ( $1: 1$ ratio) lines are guides to the eye to indicate the trend between charge generation rate and driving energy. With larger

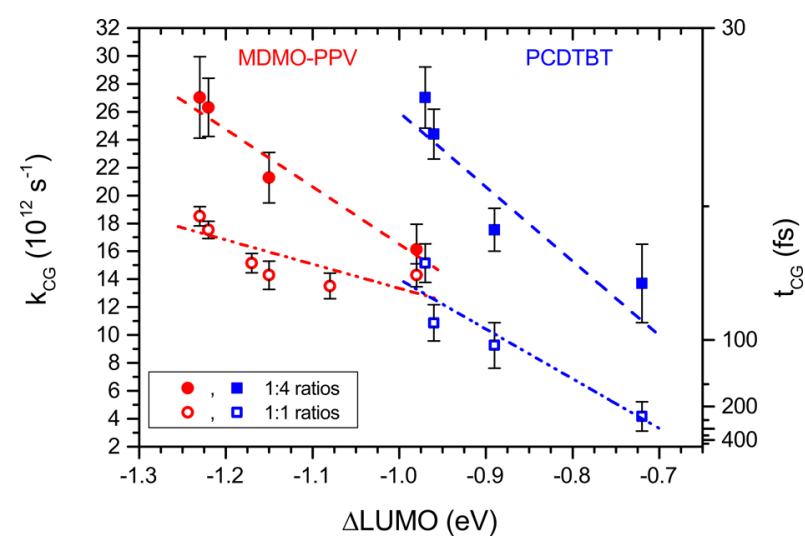

Figure 3. Charge generation rates for the different blend compositions, 1:1 (hollow symbols) and 1:4 (solid symbols) polymer/fullerene ratio. Red symbols represent the rates for blends based on MDMO-PPV, and blue symbol represent PCDTBT based blends. The error bars correspond to the standard error of the monoexponential fit. The dashed (1:4 ratio) and dotted-dashed (1:1 ratio) lines are guides to the eye how the charge generation rate is changing with driving energy.

driving energy, a faster charge generation rate is observed in the 1:1 blends. The rate increases up to the largest driving energy in the data set. Time scales for charge generation in the 1:1 blends range between 54 and $240 \mathrm{fs}$.

For samples blended at a ratio of 1:4, time scales are in the range between 37 and 75 fs. Importantly, for each polymer:fullerene combination, the charge generation rate is higher than its corresponding 1:1 composition value and most of the rates are also higher than the fastest rate in the $1: 1$ blends corresponding to 54 fs. This means that by changing the fullerene content within the sample, charge generation can proceed almost an order of magnitude faster, despite having the same LUMO offset. This is true even for fullerenes such as ICTA, which do not pack well compared to PCBM. No "inverted" Marcus regime type behavior is observed in the data set, despite the high driving energy values reached. ${ }^{27,36,37}$

We note that the error bars given for $k_{\mathrm{CG}}$ in Figure 3 are the respective standard errors from the exponential fit. They incorporate the goodness of the fit which is mostly dependent on the signal-to-noise ratio and the noise in comparison with the peak amplitude, which leads to relatively larger errors for the PCDTBT:ICTA samples. A plot including $x$-error bars corresponding to the uncertainty of the UPS measurement can be found in Figure S4.1.

Furthermore, we note that the rates of charge generation measured here correspond to only the initial ultrafast phase of charge generation, arising from excitons photogenerated directly near heterojunctions (as discussed above), which constitute the bulk of the photo excitations in the system. However, a small fraction of excitons may be generated in a pure polymer phase which first need to diffuse to the donor/ acceptor interface and then undergo $\mathrm{CT}$, giving rise to a slower picosecond charge-transfer rate, as has been observed previously. ${ }^{38-40}$ On the time scales measured here (less than 3 ps, see, e.g., Figure 2) these processes do not contribute to the observed responses. We therefore assign the difference of ultrafast CT rates to the different electronic coupling between the polymer donor and fullerene acceptor, as energetics and fullerene cluster size are varied. This result shows that while driving energy is an important parameter controlling the rate of $\mathrm{CT}$, having aggregated fullerene and hence access to a large 

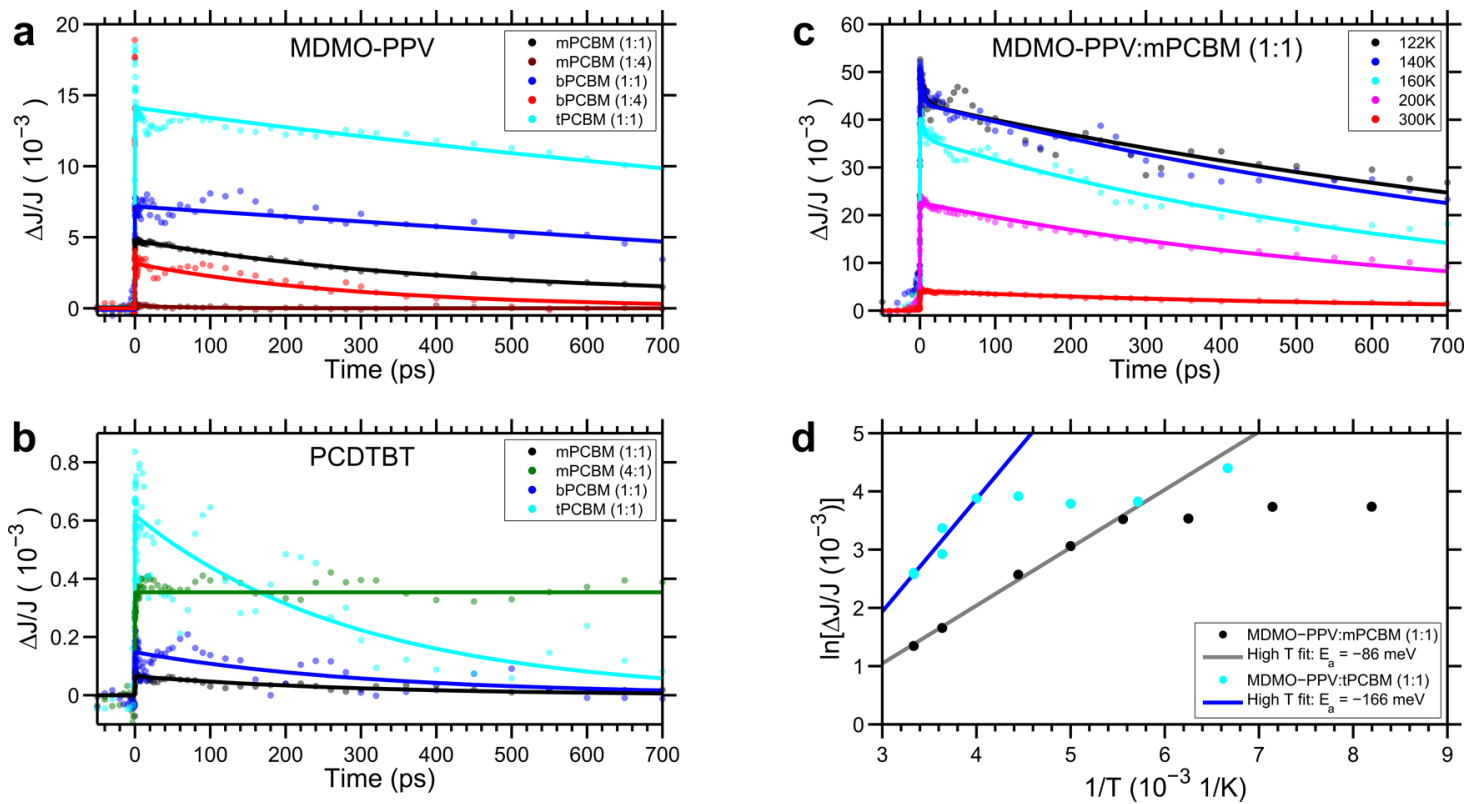

Figure 4. Pump-push-photocurrent (PPPc) kinetics for (a) MDMO-PPV:fullerene and (b) PCDTBT:fullerene blend. (c) Temperature dependent PPPc transients for MDMO-PPV:mPCBM (1:1) blends for temperatures between 122 and $300 \mathrm{~K}$ (full set in Figure S5.2a). (d) PPPc values at 40 ps for MDMO-PPV:mPCBM and tPCBM (1:1) blends in an Arrhenius plot. The solid line indicates the trend along high temperatures, yielding an activation energy of 86 and $166 \mathrm{meV}$ for the CT state detrapping in MDMO-PPVmPCBM and tPCBM blends, respectively. In all plots, the influence of the push pulse $(\Delta J)$ is normalized to the simultaneously acquired photocurrent of the device illuminated by only the pump pulse.

number of states to couple the electron transfer process is a more important factor.

In order to investigate the nature and properties of the CTSs formed by electron transfer and how they depend on the different materials and aggregations, we perform pump-pushphotocurrent $(\mathrm{PPPc})$ experiments. ${ }^{22,41}$ Here, a visible $540 \mathrm{~nm}$ pump pulse generates excitons within a working OPV device. Following CT at the heterojunction, a certain fraction of the excitons will give rise to strongly bound CTSs which will not dissociate to free charges and hence are a loss pathway. Therefore, CS and formation of bound CTSs are in direct competition. Here, we dissociate these strongly bound CTSs with a time-delayed $2000 \mathrm{~nm}(0.62 \mathrm{eV})$ infrared "push" pulse. This wavelength corresponds to the low-energy absorption feature of hole-polarons, exciting localized hole-polarons within the bound CT state to a higher-lying band-like delocalized state, as has been described previously. ${ }^{22}$ For free holepolarons, i.e., those not bound as CTSs, this excitation is followed by rapid thermalisation and there is no overall effect on device photocurrent. On the other hand, for hole-polarons bound within CTSs the push pulse may help to overcome electron-hole interaction, leading to additional free charges in the system. These additional charges contribute to extra photocurrent of the device $(\Delta J)$ and hence the effect of the push-pulse can be measured via a lock-in measurement of the photocurrent from the device $(J)$. Varying the delay between the pump and push pulse allows for the population of strongly bound CTSs to be tracked as a function of time. Importantly, the PPPc technique allows a selective probe of bound CTSs, and is not sensitive to free charges, as is the case for PP spectroscopy. We note that the PPPc results do not reveal the mechanism by which free charges are formed, thermal activation of CTS on longer time scales or ultrafast separation via delocalized states. The measurement only reports the population of bound states formed. Furthermore, the push pulse is not generating CTSs from the ground state as the push energy of $0.62 \mathrm{eV}$ is much smaller than the CTS energy of MDMO-PPV:mPCBM (1:1) at around $1.4 \mathrm{eV}$ (see Figure S1.2) or comparable polymers in the literature. ${ }^{42}$ With pushing the hole polaron as part of the CT exciton, changing the fullerene also does not significantly influence the absorption cross-section of the polaron and therefore a comparison across the set is valid and all film related variations are addressed within the normalization of $\Delta J / J$.

PPPc kinetics show that polymer blends with tris-PCBM exhibit the largest $\Delta J / J$ response to the push pulse, with smaller responses in bis- and mono-PCBM blends (Figure $4 \mathrm{a}$ and $\mathrm{b}$ ). When varying composition, samples with more fullerene content show smaller responses. This suggests that samples with larger and better aggregated fullerene clusters form fewer strongly bound CTSs following exciton dissociation, i.e., generation of free charges following photoexcitation is more efficient in these systems. We note that, in all the cases presented here, the amount of fullerene in the system is more than sufficient to form percolating networks allowing for electron transport. Thus, the cause of CTS formation is not lack of availability of a suitable path to the electrode, but rather an inability to overcome Coulomb attraction following CT.

To gain more insight on the nature of the bound CTS, we perform temperature dependent PPPc measurements on MDMO-PPV:mPCBM (1:1) and MDMO-PPV:tPCBM (1:1) blends, which both show a large fraction of bound CTSs, as judged from Figure 4a. When lowering the temperature from 300 to 122 and $150 \mathrm{~K}$, respectively, we observe an increase in $\Delta J / J$ by almost one order of magnitude in the MDMOPPV:mPCBM (1:1) blend (Figure 4c, full set in Figure S5.2a) and MDMO-PPV:tPCBM (1:1) blends (Figure S5.2b). This implies that the fraction of strongly bound CTSs increases as temperature is decreased. We note that when assuming the initial charge generation process to be temperature independent, ${ }^{43}$ this increase in CTSs is likely to be due to a lowered probability to overcome an energy barrier between CT and CS 
states as reported previously. ${ }^{16}$ Placing the values of $\Delta J / J$ at short delay times (40 ps) in an Arrhenius plot, we find that for temperatures above $\sim 200 \mathrm{~K}$, the PPPc responses show clear temperature dependence which can be described by a chargeseparation energy barrier $\left(E_{\mathrm{a}}\right)$ of $-86 \mathrm{meV}$ for MDMOPPV:mPCBM and $-166 \mathrm{meV}$ for MDMO-PPV:tPCBM. These high activation energies (3-5 times $k T$ at room temperature) provide a clear explanation for why these states remain bound as observed by Barker et al., ${ }^{6}$ therefore reducing the photocurrent yield of the device. At lower temperature, the temperature dependence changes which may be related to the transition from thermal activation into the nuclear tunnelling regime. ${ }^{44,45}$ We note that thermal activation can be clearly observed only in the "inefficient" 1:1 photovoltaic blends where delocalized states may not be readily available due to the smaller fullerene cluster size. In contrast, in efficient blends, charge separation is found to be temperature independent. ${ }^{20}$

These results represent the first direct measurements of the efficiency of ultrafast charge separation as a function of temperature, and thus the first direct measurement of CT binding energies that are deconvoluted from later time recombination and charge transport processes. From the presented data we find CTS lifetimes of less than 1 ns for MDMO-PPV:mPCBM. This lifetime is short and would not appear to be sufficient to allow the CTSs to overcome the 100$150 \mathrm{meV}$ barrier which we measure via temperature dependent pump-push experiments.

\section{DISCUSSION}

In this study we have used two ultrafast spectroscopic techniques, PP and PPPc to probe the rate of CT and efficiency of CS in model polymer-fullerene systems as a function of driving energy, fullerene aggregate size and packing. The results presented in Figure 3 show that while driving energy does have an influence on the rate of CT, the influence of fullerene aggregate size and packing are much more crucial. Indeed, keeping the same driving energy, the rate of CT can be increased by almost 3-fold by changing the fullerene cluster size. Similarly, the efficiency of CS (Figure 4) is much more sensitive to fullerene aggregates than to driving energy. Indeed, the similar trend observed for both processes provides strong evidence that both the rate of CT and CS are controlled by the same parameters, namely, fullerene aggregate size and packing.

The picture that emerges from these results is one that cannot be rationalized via simple concepts such as driving energy and the use of modified Marcus models that consider only point like charges to predict charge generation rates based on the energetics of isolated molecules. The dependence of the rate of electron transfer on driving energy for instance, does not correspond to what Marcus theory would predict. The lifetimes of CTSs, measured using pump-push spectroscopy, are not long enough to allow thermal activation over the $100-150 \mathrm{meV}$ barriers observed. This suggests that the dissociation of CTSs is not governed simply by hopping transport as within the Onsager-Braun framework.

Our results are more consistent with models that explicitly consider the role of fullerene aggregate size, packing and corresponding delocalization of charges. It has been proposed that increasing fullerene cluster size leads to a larger density of delocalized states on the fullerene cluster and therefore a higher rate of charge generation $k_{\mathrm{CG}}$ (step 1, Figure 5), ${ }^{43}$ which is what we observe in Figure 3. This could drive fast and efficient charge generation by directly injecting electrons into band like

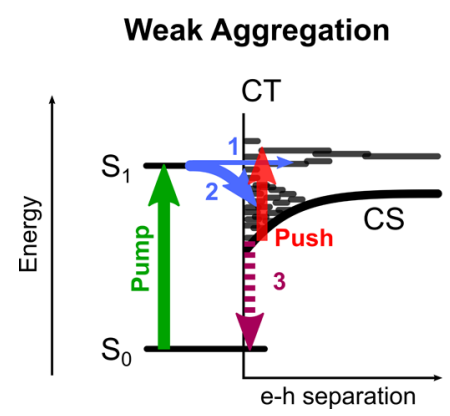

Strong Aggregation

Figure 5. Schematic of electronic states in an organic solar cell and different processes happening after excitation. The excited state $\left(S_{1}\right)$ can be directly separated (1) or form charge transfer (CT) states (2) which serve as a main recombination pathway (3). The more states are accessible in the charge separated state, the higher $k_{\mathrm{CG}}$ and the more efficient the initial charge generation step, outcompeting the formation of strongly bound CTSs.

states, kinetically outcompeting the formation of interfacially bound CTSs (step 2, Figure 5), which are a precursor state for recombination (step 3, Figure 5).

This is consistent with our SAXS/WAXS data (see section S3) where we find the largest aggregates for monofunctionalized fullerenes, while bis- and tris-fullerene adducts form smaller clusters. This limits delocalization of electrons, thereby suppressing the CT and CS rates (see Figure 3). We note that such crystallization behavior is in agreement with recently reported calculations suggesting that fewer functional side groups within the fullerene derivate promote an ordered molecular packing. ${ }^{34}$

Our results suggest that very little driving energy is required at the heterojunction to dissociate excitons and generate free charges. This is also consistent with reports of polymer:fullerene blends exhibiting internal quantum efficiencies higher than $90 \%$ even when the sub-band gap CTSs are directly excited. $^{42,46}$ Our results are also consistent with recent reports on entropic gain driving charge separation ${ }^{47,48}$ as larger fullerene clusters exhibit a higher number of accessible states. ${ }^{43}$ While entropy had been mentioned as an underestimated factor before, ${ }^{10,49}$ the absolute effect of entropic gain including delocalization needs to be studied further. ${ }^{50,51}$ The most critical aspect of designing OPV systems should thus be control over nanoscale morphology to achieve the optimal molecular aggregation and provide access to delocalized states, which control the rate of ultrafast CT and charge separation. This should allow OPVs with reduced energetic losses associated with charge generation and thus boost $V_{\mathrm{OC}}$ and achieve higher power conversion efficiencies. More generally, our results on driving energy and state availability are applicable to any solid state system where strongly bound excitons need to be dissociated, such as hybrid organic-inorganic PVs or artificial photosynthetic systems for solar fuel generation. In all these systems, increasing state availability for CT, by controlling nanoscale morphology and aggregate size, should allow for energetic losses associated with efficient charge generation to be offset.

\section{METHODS}

Sample Preparation. PCDTBT, ICMA, ICBA, and ICTA were purchased from 1-material, $\mathrm{PCBM}$, bis-PCBM, and tris-PCBM were purchased from Solenne BV, and MDMO-PPV was purchased from Merck (lisicon PDO-121) and all materials were used as received. 
Films were spun from chlorobenzene (MDMO-PPV blends) or ortho-dichlorobenzene (PCDTBT blends) with $10 \mathrm{mg} / \mathrm{mL}$ for $1: 1$ blends and $15 \mathrm{mg} / \mathrm{mL}$ for $1: 4$ blends, respectively. Films were prepared on ca. $130 \mu \mathrm{m}$ thick glass substrates which were cleaned by sonication in acetone and isopropyl alcohol and exposure to $\mathrm{O}_{2}$ plasma for $10 \mathrm{~min}$ each. Substrates were subsequently brought in an oxygen and water free glovebox $\left(\mathrm{O}_{2}<5 \mathrm{ppm}, \mathrm{H}_{2} \mathrm{O}<1 \mathrm{ppm}\right)$ and spun at $1500 \mathrm{rpm}$ for 60 or $120 \mathrm{~s}$ when using chlorobenzene or dichlorobenzene, respectively. Films were encapsulated in the glovebox using carbon tape as a spacer to a second glass slide and sealing the edges with epoxy resin.

Solar cell devices were fabricated on ITO substrates, cleaned as described above. PEDOT:PSS (P VP Al 4083, Heraeus) was filtered, spun on the ITO at $4000 \mathrm{rpm}$, and then sintered at $150{ }^{\circ} \mathrm{C}$ for $30 \mathrm{~min}$. PCDTBT:fullerene films were made from $30 \mathrm{mg} / \mathrm{mL}$ in orthodichlorobenzene solution except the PCDTBT:mPCBM (4:1) blend which was spun from $20 \mathrm{mg} / \mathrm{mL}$ concentration. MDMO-PPV:fullerene blend layers were fabricated from $15 \mathrm{mg} / \mathrm{mL}$ solved in chlorobenzene. All films were spun at $1500 \mathrm{rpm}$ under the same conditions as the films described above, an aluminum electrode was evaporated on top and the devices subsequently encapsulated.

Ultrafast Transient Absorption Spectroscopy (TA). Transient absorption spectra were acquired using a $1 \mathrm{kHz}$ regenerative Ti:sapphire amplifier (Solstice, Spectra-Physics), seeding two homebuilt broadband noncollinear optical parametric amplifiers (NOPAs). One NOPA was used as a broadband visible probe while the other NOPA was used as the pump source, tuned for an output of 520 to $630 \mathrm{~nm}$ and yielding sub-40 fs pulses with $4 \mu \mathrm{J} / \mathrm{cm}^{2}$ pulse energy after compression using a pair of dielectric chirped mirrors (Layertec 109811) and sub-20 fs pulses with a pulse energy of $40 \mu \mathrm{J} / \mathrm{cm}^{2}$ after compression using a combination of dielectric chirped mirrors (Femtolasers GSM032) and a spatial light modulator applying multiphoton intrapulse interference phase scan (MIIPS, Biophotonics femtoJock). The probe light was split into two separate beams, one probe and one reference beam. Both got dispersed with a grating spectrograph (Shamrock SR-303i, Andor Technology) and measured simultaneously with a CCD detector array each (Entwicklungsbüro Stresing). Here, the reference is not subject to the pump beam and is therefore used to correct for shot-to-shot fluctuations in the system.

Pump-Push-Photocurrent (PPPc) Spectroscopy. In the PPPc experiment, the output of a regenerative $1 \mathrm{kHz}$ Ti:sapphire amplifier system (Solstice, Spectra Physics, $800 \mathrm{~nm}, 100$ fs pulse duration, 3.5 $\mathrm{mJ}$ per pulse) was split into two parts. One part was to pump a broadband noncollinear optical amplifier to generate visible pump pulses ( $100 \mathrm{fs}$ pulse duration, $2.30 \mathrm{eV}$ photon energy). The other part was used to generate mid-IR push pulses by pumping an optical parametric amplifier (TOPAS, Light Conversion, 150 fs pulse duration, $0.62 \mathrm{eV}$ photon energy).

During the experiments, the devices were measured at short-circuit conditions with pump and push pulses focusing on a $0.5 \mathrm{~mm}^{2}$ spot on the working devices. The delay between pump and push pulse is generated via a mechanical delay stage. The current $J$ induced by the pump pulse $(1 \mathrm{kHz}$, in the order of $1 \mathrm{~nJ})$ was detected using a lock-in amplifier (Stanford Instruments) working in current mode. The $5 \mu \mathrm{J}$ push pulse was mechanically modulated at $370 \mathrm{~Hz}$ and the extra photocurrent generated, $\Delta \mathrm{J}$, was detected by the same lock-in amplifier locked to the chopper frequency. The fractional change in photocurrent, $\Delta J / J$, can thus be calculated.

Photothermal Deflection Spectroscopy (PDS). PDS samples were prepared by spin-coating PCDTBT and MDMO-PPV from 10 $\mathrm{mg} / \mathrm{mL}$ solution in ortho-dichlorobenzene and chlorobenzene, respectively, and the fullerenes from $40 \mathrm{mg} / \mathrm{mL}$ solution in chloroform on water free quartz substrates (Spectrosil).

PDS is a highly sensitive absorption measurement technique. For the measurements, a monochromatic pump beam is shone on to the sample (film on Quartz substrate), which on absorption produces a thermal gradient near the sample surface via nonradiative relaxation induced heating. This results in a refractive index gradient in front of the sample surface, which is further enhanced by immersing the sample in an inert liquid FC-72 Fluorinert (3 M Company) which has a high refractive index change per unit change in temperature. A fixed wavelength $\mathrm{CW}$ probe laser beam is passed through this refractive index gradient producing a deflection, which is proportional to the light absorbed in the sample at that particular wavelength. This deflection in laser beam is further detected by a photodiode and lockin amplifier combination. Scanning through different wavelengths gives us the complete absorption spectra.

Wide/Small Angle X-ray Scattering (WAXS/SAXS). SAXS and WAXS measurements of the bulk material were simultaneously carried out at the SAXS beamline of the Australian Synchrotron using a Pilatus $1 \mathrm{M}$ and a Pilatus 200k detector. Beam energy and distance between sample and detector distances were chosen so that SAXS and WAXS spectra showed an overlap between both signals and they were afterward joined together at $q=0.73 \AA^{-1}$.

Ultraviolet Photoelectron Spectroscopy (UPS). Samples for UPS measurements were prepared by spin-coating PCDTBT and MDMO-PPV from $10 \mathrm{mg} / \mathrm{mL}$ solution in ortho-dichlorobenzene and chlorobenzene, respectively, and the fullerenes from $20 \mathrm{mg} / \mathrm{mL}$ solution in chlorobenzene onto gold-coated silicon wafer substrates. The samples were subsequently transferred to an ultrahigh vacuum (UHV) chamber (ESCALAB 250Xi) in which UPS measurements were carried out using a XR6 monochromated $\mathrm{Al} \mathrm{K} \alpha \mathrm{X}$-ray source $(h \nu$ $=1486.6 \mathrm{eV}$ ) with a $900 \mu \mathrm{m}$ spot size and $21.22 \mathrm{eV}$ pass energy. Data analysis to extract the HOMO levels was performed using previously reported algorithms. ${ }^{52}$

\section{ASSOCIATED CONTENT}

\section{Supporting Information}

The Supporting Information is available free of charge on the ACS Publications website at DOI: 10.1021/jacs.6b05131.

UV-vis and PL spectra for the polymers used and PCBM, PL of the CT state of MDMO-PPV:mPCBM, details of the determination of energy levels for the different materials including UPS and PDS data and comparison with the literature, analysis of SAXS/WAXS data of the different polymer:fullerene blends and neat materials, TA kinetics used for determination of CT times, comparison of TA kinetics of different species and at different fluences, and full sets of temperature dependent PPPc measurements (PDF)

\section{AUTHOR INFORMATION}

\section{Corresponding Authors}

*ar525@cam.ac.uk

*rhf10@cam.ac.uk

\section{Notes}

The authors declare no competing financial interest.

Measurement Data: Additional data related to this publication is available at the University of Cambridge data repository at http://dx.doi.org/10.17863/CAM.1183.

\section{ACKNOWLEDGMENTS}

This work was supported by the Engineering and Physical Sciences Research Council (EPSRC) and the Winton Programme for the Physics of Sustainability. A.C.J. thanks the University of Cambridge for funding (CHESS). J.Z. thanks the China Scholarship Council for financial support for $\mathrm{PhD}$ studies. S.H. thanks the framework project Soltech for funding. We thank Adam Brown for conducting the UPS measurements. Synchrotron measurements were undertaken on the SAXS beamline at the Australian Synchrotron, Victoria, Australia, and we acknowledge the help of Niraj Lal with the measurements. 


\section{REFERENCES}

(1) Tang, C. W. Appl. Phys. Lett. 1986, 48, 183.

(2) Sariciftci, N. S.; Smilowitz, L.; Heeger, A. J.; Wudl, F. Science 1992, 258, 1474-1476.

(3) Halls, J. J. M.; Walsh, C. A.; Greenham, N. C.; Marseglia, E. A.; Friend, R. H.; Moratti, S. C.; Holmes, A. B. Nature 1995, 376, 498.

(4) Yu, G.; Heeger, A. J. J. Appl. Phys. 1995, 78, 4510.

(5) Shaheen, S. E.; Brabec, C. J.; Sariciftci, N. S.; Padinger, F.; Fromherz, T.; Hummelen, J. C. Appl. Phys. Lett. 2001, 78, 841.

(6) Mihailetchi, V. D.; Koster, L. J. A.; Blom, P. W. M.; Melzer, C.; de Boer, B.; van Duren, J. K. J.; Janssen, R. A. J. Adv. Funct. Mater. 2005, 15,795 .

(7) Veldman, D.; Meskers, S. C. J.; Janssen, R. A. J. Adv. Funct. Mater. 2009, 19, 1939.

(8) Scharber, M. C.; Mühlbacher, D.; Koppe, M.; Denk, P.; Waldauf, C.; Heeger, A. J.; Brabec, C. J. Adv. Mater. 2006, 18, 789.

(9) Pfadler, T.; Coric, M.; Palumbiny, C. M.; Jakowetz, A. C.; Strunk, K.-P.; Dorman, J. A.; Ehrenreich, P.; Wang, C.; Hexemer, A.; Png, R.Q.; Ho, P. K. H.; Weickert, J.; Schmidt-Mende, L. ACS Nano 2014, 8, 12397.

(10) Clarke, T. M.; Durrant, J. R. Chem. Rev. 2010, 110, 6736.

(11) Bässler, H.; Köhler, A. Phys. Chem. Chem. Phys. 2015, 17, 28451.

(12) Janssen, R. A. J.; Nelson, J. Adv. Mater. 2013, 25, 1847.

(13) Vandewal, K.; Ma, Z.; Bergqvist, J.; Tang, Z.; Wang, E.; Henriksson, P.; Tvingstedt, K.; Andersson, M. R.; Zhang, F.; Inganäs, O. Adv. Funct. Mater. 2012, 22, 3480.

(14) Pensack, R. D.; Asbury, J. B. J. Phys. Chem. Lett. 2010, 1, 2255.

(15) Zusan, A.; Vandewal, K.; Allendorf, B.; Hansen, N. H.; Pflaum, J.; Salleo, A.; Dyakonov, V.; Deibel, C. Adv. Energy Mater. 2014, 4, 1400922.

(16) Barker, A. J.; Chen, K.; Hodgkiss, J. M. J. Am. Chem. Soc. 2014, 136, 12018

(17) Troisi, A. Faraday Discuss. 2013, 163, 377.

(18) Tamura, H.; Burghardt, I. J. Am. Chem. Soc. 2013, 135, 16364.

(19) Etzold, F.; Howard, I. A.; Mauer, R.; Meister, M.; Kim, T.-D.;

Lee, K.-S.; Baek, N. S.; Laquai, F. J. Am. Chem. Soc. 2011, 133, 9469.

(20) Gelinas, S.; Rao, A.; Kumar, A.; Smith, S. L.; Chin, A. W.; Clark, J.; van der Poll, T. S.; Bazan, G. C.; Friend, R. H. Science 2014, 343, 512 .

(21) Vithanage, D. A.; Devižis, A.; Abramavičius, V.; Infahsaeng, Y.; Abramavičius, D.; MacKenzie, R. C. I.; Keivanidis, P. E.; Yartsev, A.; Hertel, D.; Nelson, J.; Sundström, V.; Gulbinas, V. Nat. Commun. 2013, 4, 2334.

(22) Bakulin, A. A.; Rao, A.; Pavelyev, V. G.; van Loosdrecht, P. H M.; Pshenichnikov, M. S.; Niedzialek, D.; Cornil, J.; Beljonne, D.; Friend, R. H. Science 2012, 335, 1340.

(23) Few, S.; Frost, J. M.; Nelson, J. Phys. Chem. Chem. Phys. 2015, $17,2311$.

(24) Pavelyev, V. G.; Parashchuk, O. D.; Krompiec, M.; Orekhova, T. V.; Perepichka, I. F.; van Loosdrecht, P. H. M.; Paraschuk, D. Y.; Pshenichnikov, M. S. J. Phys. Chem. C 2014, 118, 30291-30301.

(25) Bakulin, A. A.; Dimitrov, S. D.; Rao, A.; Chow, P. C. Y.; Nielsen, C. B.; Schroeder, B. C.; McCulloch, I.; Bakker, H. J.; Durrant, J. R.; Friend, R. H. J. Phys. Chem. Lett. 2013, 4, 209.

(26) Ohkita, H.; Cook, S.; Astuti, Y.; Duffy, W.; Tierney, S.; Zhang, W.; Heeney, M.; McCulloch, I.; Nelson, J.; Bradley, D. D. C.; Durrant, J. R. J. Am. Chem. Soc. 2008, 130, 3030.

(27) Coffey, D. C.; Larson, B. W.; Hains, A. W.; Whitaker, J. B.; Kopidakis, N.; Boltalina, O. V.; Strauss, S. H.; Rumbles, G. J. Phys. Chem. C 2012, 116, 8916.

(28) Faist, M. A.; Shoaee, S.; Tuladhar, S.; Dibb, G. F. A.; Foster, S.; Gong, W.; Kirchartz, T.; Bradley, D. D. C.; Durrant, J. R.; Nelson, J. Adv. Energy Mater. 2013, 3, 744.

(29) Faist, M. A.; Kirchartz, T.; Gong, W.; Ashraf, R. S.; McCulloch, I.; de Mello, J. C.; Ekins-Daukes, N. J.; Bradley, D. D. C.; Nelson, J. J. Am. Chem. Soc. 2012, 134, 685.

(30) Frost, J. M.; Faist, M. A.; Nelson, J. Adv. Mater. 2010, 22, 4881.

(31) Miller, N. C.; Cho, E.; Gysel, R.; Risko, C.; Coropceanu, V.; Miller, C. E.; Sweetnam, S.; Sellinger, A.; Heeney, M.; McCulloch, I.;
Brédas, J.-L.; Toney, M. F.; McGehee, M. D. Adv. Energy Mater. 2012 2, 1208 .

(32) Cates, N. C.; Gysel, R.; Dahl, J. E. P.; Sellinger, A.; McGehee, M. D. Chem. Mater. 2010, 22, 3543.

(33) Beiley, Z. M.; Hoke, E. T.; Noriega, R.; Dacuña, J.; Burkhard, G. F.; Bartelt, J. A.; Salleo, A.; Toney, M. F.; McGehee, M. D. Adv. Energy Mater. 2011, 1, 954.

(34) Savoie, B. M.; Kohlstedt, K. L.; Jackson, N. E.; Chen, L. X.; Olvera de la Cruz, M.; Schatz, G. C.; Marks, T. J.; Ratner, M. A. Proc. Natl. Acad. Sci. U. S. A. 2014, 111, 10055.

(35) Lanzani, G.; Cerullo, G.; Brabec, C.; Sariciftci, N. S. Phys. Rev. Lett. 2003, 90, 047402.

(36) Ward, A. J.; Ruseckas, A.; Kareem, M. M.; Ebenhoch, B.; Serrano, L. A.; Al-Eid, M.; Fitzpatrick, B.; Rotello, V. M.; Cooke, G.; Samuel, I. D. W. Adv. Mater. 2015, 27, 2496.

(37) Marcus, R. A. Discuss. Faraday Soc. 1960, 29, 21.

(38) Cook, S.; Katoh, R.; Furube, A. J. Phys. Chem. C 2009, 113, 2547.

(39) Bakulin, A. A.; Hummelen, J. C.; Pshenichnikov, M. S.; Van Loosdrecht, P. H. M. Adv. Funct. Mater. 2010, 20, 1653.

(40) Kozlov, O. V.; de Haan, F.; Kerner, R. A.; Rand, B. P.; Cheyns, D.; Pshenichnikov, M. S. Phys. Rev. Lett. 2016, 116, 057402.

(41) Bakulin, A. A.; Silva, C.; Vella, E. J. Phys. Chem. Lett. 2016, 7, 250-258.

(42) Vandewal, K.; Albrecht, S.; Hoke, E. T.; Graham, K. R.; Widmer, J.; Douglas, J. D.; Schubert, M.; Mateker, W. R.; Bloking, J. T.; Burkhard, G. F.; Sellinger, A.; Fréchet, J. M. J.; Amassian, A.; Riede, M. K.; McGehee, M. D.; Neher, D.; Salleo, A. Nat. Mater. 2014, 13, 63.

(43) Savoie, B. M.; Rao, A.; Bakulin, A. A.; Gelinas, S.; Movaghar, B.; Friend, R. H.; Marks, T. J.; Ratner, M. A. J. Am. Chem. Soc. 2014, 136, 2876.

(44) Asadi, K.; Kronemeijer, A. J.; Cramer, T.; Koster, L. J. A.; Blom, P. W. M.; de Leeuw, D. M. Nat. Commun. 2013, 4, 1710.

(45) Bakulin, A. A.; Lovrincic, R.; Yu, X.; Selig, O.; Bakker, H. J.; Rezus, Y. L. a; Nayak, P. K.; Fonari, A.; Coropceanu, V.; Brédas, J.-L.; Cahen, D. Nat. Commun. 2015, 6, 7880.

(46) Albrecht, S.; Vandewal, K.; Tumbleston, J. R.; Fischer, F. S. U.; Douglas, J. D.; Fréchet, J. M. J.; Ludwigs, S.; Ade, H.; Salleo, A.; Neher, D. Adv. Mater. 2014, 26, 2533.

(47) Gao, F.; Tress, W.; Wang, J.; Inganäs, O. Phys. Rev. Lett. 2015, $114,128701$.

(48) Monahan, N. R.; Williams, K. W.; Kumar, B.; Nuckolls, C.; Zhu, X.-Y. Phys. Rev. Lett. 2015, 114, 247003.

(49) Gregg, B. A. J. Phys. Chem. Lett. 2011, 2, 3013.

(50) Hood, S. N.; Kassal, I. 2016, arXiv:1603.06304. arXiv.org e-Print archive. http://arxiv.org/abs/1603.06304.

(51) Ono, S.; Ohno, K. 2016, arXiv:1607.07946. arXiv.org e-Print archive. http://arxiv.org/abs/1607.07946.

(52) Böhm, M. L.; Jellicoe, T. C.; Rivett, J. P. H.; Sadhanala, A.; Davis, N. J. L. K.; Morgenstern, F. S. F.; Gödel, K. C.; Govindasamy, J.; Benson, C. G. M.; Greenham, N. C.; Ehrler, B. J. Phys. Chem. Lett. 2015, 6, 3510 . 